\title{
Quantum Erasure as Quantum Predestination
}

\author{
Terry B. Bollinger (1) 1 \\ ${ }^{1}$ Apabistia Research, Ashburn, VA, USA 20147
}

(Incepted 31 October 2021; Published 31 October 2021; Tweaked 2021-11-01.01:29)*

\begin{abstract}
As indicated by the name "quantum erasure," the most common interpretation of certain classes of delayed choice quantum experiments is that they, in some fashion, erase or undo past decisions. Unfortunately, this interpretation cannot be correct since the past decisions were already classically and irreversibly captured as recorded information or datums. A datum is information that, through temporal entanglement, constrains future events. The correct interpretation of such experiments is stranger than erasure: Recordings made early in such quantum experiments predestine choices made later through arbitrarily complex and often human-scale classical choices. Since this process of quantum predestination occurs only within the future light cone of datum creation, another (possibly) less radical way to interpret such experiments is that time is multiscale, granular, and impossible to define outside of the quantum state of the entities involved. The continuum time abstraction is not compatible with this view.
\end{abstract}

DOI: https://doi.org/10.48034/20211031

This is about: Quantum erasure, Quantum entanglement

\section{INTRODUCTION}

In her October 2021 Backreaction video "The delayed choice quantum eraser, debunked" [1], Sabine Hossenfelder explains why the early recording of an interference pattern in a quantum erasure experiment precludes any future erasure of that result. One striking consequence of Dr. Hossenfelder's observation is that if early events in a quantum erasure experiment are causally irreversible, then all future classical events falling in the light cone of that initial recording event are predestined to occur in ways that support the initial finding. The fact that such effects remain constrained to the future light cone of the initial event enables another unsettling interpretation of this quantum predestination effect: time is both granular and multiscale. This interpretation is potentially disturbing since if it is always and the quantum state of interacting entities that determines the passage of classical time, then a simple continuum representation of time is insufficiently detailed to predict temporal evolution fully.

\section{THE HOSSENFELDER EXPERIMENT}

A slightly elaborated version of the Hossenfelder

Published by Apabistia Press under the Creative Commons Attribution 4.0 International License. You are free to redistribute this article as you choose, provided only that you preserve the author attribution, article title, journal citation, and DOI.

${ }^{*}$ This document uses Kenobi versioning. The online name always points to the most recent document version, while ob1, ob2,... suffixes indicate obsolete releases of the document. quantum erasure experiment is in Figure 1. The Hossenfelder version of quantum erasure is helpful analytically primarily by placing the entangled views of each photon at the ends of two loops that can vary vastly in length. The elaboration in this figure emphasizes just that point: For any given photon, the horizontal length can be made arbitrarily longer than the vertical one, or viceversa. The other simple point of this variant is nothing more than the observation that the shorter vertical observation is necessarily classical, producing a historical record of the interference pattern achieved that is no longer subject to quantum indeterminacy. Unless one chooses to disregard causality entirely, the speed with which current networking methods can record and spread makes the result as classical as the words you are now reading.

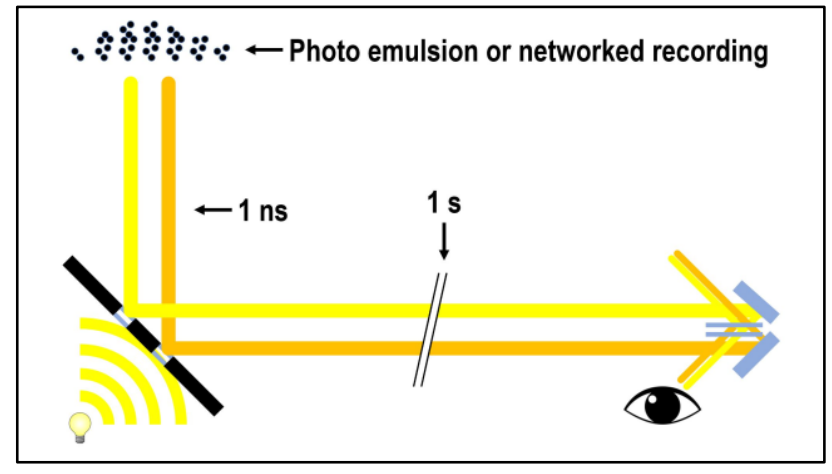

FIG. 1. The Hossenfelder quantum erasure experiment. This figure elaborates slightly on the Hossenfelder [1] figure shown at 7 minutes $33 \mathrm{~s}$ (https://youtu.be/RQv5CVELG3U?t=7m33s). 
See the Hossenfelder video for a description of the principles of how partial and complete reflections combine to create the quantum erasure experiment. Hossenfelder elaborates on how finding which way the photon goes does not make it more particle-like but instead creates a broader wave-like distribution of probabilities for the next sighting of the particle. For this paper, the critical detail of the Hossenfelder experiment is this: The particular class of interference pattern - detailed or smooth - recorded one nanosecond after the launch of a photon necessarily and unavoidably depends on the observation configuration occurring one second after photon launch on the horizontal branch of the figure. One such configuration is shown here by an observing eye - another classical recorder - on the lower of the two merged outputs.

\section{PROCEDURE}

Add a randomized observation-mode selector to the one-second branch. Send out photons at sufficiently low luminosity to ensure that individual results are associated with high confidence to the randomized observation configurations on the one-second branch. Collect enough observations to identify the one-nanosecond interference patterns for each of the possible one-second observation settings. Both theory and experimentation indicate that the final data sets should show that the settings on the onesecond branch determine the interference patterns seen on the one-nanosecond branch.

\section{THE PROBLEM WITH ERASURE}

As indicated directly by the name of the experiments, the dependence of the (in this case) one-nanosecond outcomes on the later (delayed) one-second observation choices is typically called erasure of the earlier results.

The difficulty of the erasure interpretation of the results is that the observations made at one nanosecond are no longer part of the quantum time domain in which such ambiguities can exist. Once replicable information is created in the experiment, classical causality requires that this information remains intact. The one-nanosecond sensor could, for example, go instantly into a global network or a massive database with multiple impacts on historical events in the classical world. Any other interpretation of such datums, that is, of classical data that remains entangled with future quantum events, results in sufficient undermining of the concept of reality to make scientific analysis pointless. There is no value in this path.

As pointed out by Hossenfelder in her video, the resolution is to accept the reality of the one-nanosecond datums in all subsequent events. The datum outcomes thus are functions not from a single moment in time but over an extended interval of spacetime in which events taking place at one second are just as experimentally impactful as those taking place at one nanosecond.

\section{QUANTUM PREDESTINATION}

While such ideas of the dependency of the present on events in the future are by no means unprecedented in the history of physics [2] [3], they are nonetheless unnerving due to the complexity and unpredictability of the classical world. However, if one accepts that quantum-entangled classical datums in such experiments are predictors of subsequent classical configuration data, the phrase quantum predestination becomes more apt terminology.

Quantum predestination occurs whenever information recorded early in a quantum event occurring over a large expanse of spacetime requires later classical events ones without any apparent connection to the earlier event - to occur in a fashion that maintains the predictions of the quantum-entangled datums of information.

Note that invoking the block universe concept does not resolve quantum predestination since it does not explain how such resolutions occur. Instead, the block universe is a faith assertion that somehow, the universe figures out the needed resolutions for us. This view is not a scientific position because it permits no serious analysis of the quantum predestination conundrum.

\section{LIGHT CONE CONSTRAINTS}

A notable feature of datums is that they only impact events within their future light cones. The light cone limit emerges due to the new post-datum wave, e.g., after the location of a particle within a small region of space, expanding no faster than the speed of light. This same feature prevents causal paradoxes in quantum predestination since it is never possible to signal the future to avoid a specific configuration already predestined by an earlier datum event.

While quantum predestination is an unavoidably mindboggling concept, the fact that it cannot occur outside of its future light cone shows it still follows definite rules and limitations. That in turn indicates that the problem lies not in quantum predestination itself, but in our understanding of how time works.

\section{A GRANULAR VIEW OF SPACETIME}

One possible path for resolving quantum predestination is to stop thinking of classical time as anything more than an emergent quantum phenomenon in which the passage of time depends on phenomena with no such limits. Instead, fundamentally timeless quantum phenomena become constrained in the presence of datums, with the consensus of such effects forming what emulates the smooth passage of time at classical scales.

Quantum predestination experiments are, in this view, examples of quantum effects where one cannot ignore the spacetime size or granularity of such essentially timeless quantum events. The evidence for such large-grained time 
components is in the predestination effect itself, in which events occurring in the distant (e.g., one-second) future light cone are just as relevant to datum creation as events in the past light cone.

While such a perspective arguably resembles a block universe perspective, the critical difference is that a granular and multiscale definition of time is inherently less precise and less data-intensive entire block universe. The future would form slowly in response to the collective constraints of earlier datums, emerging as a viable consensus of what is possible given those past constraints.

\section{CONCLUSION}

Quantum erasure is a term in need of replacement. While quantum predestination is scarier in terms of its implication for how classical physics emerges from the quantum world, it also appears to be a more accurate description of the outcomes seen in such experiments.

At a deeper level, such experiments suggest the need for a more radical interpretation of time itself. It would begin with essentially limitless quantum phenomena and show how the emergence of information, specifically of futurelimiting entangled datums, continually constraints and limits that quantum world into the world of meaningful time flow and causality in which we exist.

[1] S. Hossenfelder, "The delayed choice quantum eraser, debunked," Backreaction, 30 October 2021.

[2] J. A. Wheeler and R. P. Feynman, "Interaction with the Absorber as the Mechanism of Radiation," Reviews of Modern Physics, vol. 17, p. 157, 1945.

[3] J. A. Wheeler and R. P. Feynman, "Classical Electrodynamics in Terms of Direct Interparticle Action," Reviews of Modern Physics, vol. 21, p. 425, 1949. 